\title{
Irrationality of values of $L$-functions of Dirichlet characters
}

\author{
Stéphane Fischler
}

April 5, 2019

\begin{abstract}
In a recent paper with Sprang and Zudilin, the following result was proved: if $a$ is large enough in terms of $\varepsilon>0$, then at least $2^{(1-\varepsilon) \frac{\log a}{\log \log a}}$ values of the Riemann zeta function at odd integers between 3 and $a$ are irrational. This improves on the Ball-Rivoal theorem, that provides only $\frac{1-\varepsilon}{1+\log 2} \log a$ such irrational values - but with a stronger property: they are linearly independent over the rationals.

In the present paper we generalize this recent result to both $L$-functions of Dirichlet characters and Hurwitz zeta function. The strategy is different and less elementary: the construction is related to a Padé approximation problem, and a generalization of Shidlovsky's lemma is used to apply Siegel's linear independence criterion.

We also improve the analogue of the Ball-Rivoal theorem in this setting: we obtain $\frac{1-\varepsilon}{1+\log 2} \log a$ linearly independent values $L(f, s)$ with $s \leq a$ of a fixed parity, when $f$ is a Dirichlet character. The new point here is that the constant $1+\log 2$ does not depend on $f$.
\end{abstract}

MSC 2010: 11J72 (Primary); 11M06, 11M35, 33C20 (Secondary).

The purpose of this paper is to prove results of irrationality, or linear independence, of values of the Hurwitz $\zeta$ function or $L$-functions of Dirichlet characters. Both are generalizations of the Riemann $\zeta$ function, so we begin with a quick survey of the main results in this setting.

When $s \geq 2$ is even, $\zeta(s) \pi^{-s}$ is a non-zero rational number so that $\zeta(s)$ is transcendental. Apéry has proved [1] that $\zeta(3)$ is irrational, but there is no odd $s \geq 5$ for which $\zeta(s)$ is known to be irrational. The next breakthrough is due to Ball-Rivoal $[2,20]$ :

$$
\operatorname{dim}_{\mathbb{Q}} \operatorname{Span}_{\mathbb{Q}}(1, \zeta(3), \zeta(5), \ldots, \zeta(a)) \geq \frac{1+o(1)}{1+\log 2} \log a \text { as } a \rightarrow \infty, a \text { odd. }
$$

Here and throughout this introduction, $o(1)$ denotes any sequence that tends to 0 as $a \rightarrow \infty$. In this paper we mention only asymptotic results (namely, as $a \rightarrow \infty$ ) eventhough 
most results can be made explicit, and often refined, for small values of $a$. At last we mention the following recent result [11]:

at least $2^{(1-o(1)) \frac{\log a}{\log \log a}}$ numbers among $\zeta(3), \zeta(5), \ldots, \zeta(a)$ are irrational,

for $a$ odd, $a \rightarrow \infty$.

The natural setting to generalize these results to values of the Hurwitz $\zeta$ function or $L$-functions of Dirichlet characters is the following. Let $T \geq 1$, and $f: \mathbb{Z} \rightarrow \mathbb{C}$ be such that $f(n+T)=f(n)$ for any $n$. We assume that $f$ is not identically zero. Let $\varepsilon>0$, and $a$ be sufficiently large (in terms of $T$ and $\varepsilon$ ). For $p \in\{0,1\}$ consider the complex numbers

$$
L(f, s)=\sum_{n=1}^{\infty} \frac{f(n)}{n^{s}} \text { with } 2 \leq s \leq a \text { and } s \equiv p \bmod 2 .
$$

If $f$ is a Dirichlet character mod $T$ then these are exactly the values of the associated $L$-function.

The restriction on the parity of $s$ in $(0.2)$ is needed in some cases to get rid of powers of $\pi$. Indeed, if $f$ is a Dirichlet character then $f$ is either even (i.e., $f(-n)=f(n)$ ) or odd (i.e., $f(-n)=-f(n))$, according to whether $f(-1)$ is equal to 1 or -1 . If $s \geq 2$ has the same parity as $f$ then $L(f, s) \pi^{-s}$ is a non-zero algebraic number (see for instance [18, Chapter VII, §2]) so that the numbers $L(f, s)$ for $s$ with this parity are linearly independent over $\overline{\mathbb{Q}}$. Moreover, for any periodic map $f: \mathbb{Z} \rightarrow \mathbb{Q}$ which is either even or odd (and not identically zero), we also have $L(f, s) \pi^{-s} \in \overline{\mathbb{Q}}^{*}$ when $s$ and $f$ have the same parity (see [12]). In these situations, we prove new results on the numbers (0.2) only when $p$ and $f$ have opposite parities.

An interesting case where (in general) $f$ is neither odd nor even is the following. Given $u \in\{1, \ldots, T-1\}$ we define $f$ by $f(n)=1$ if $n \equiv u \bmod T$, and $f(n)=0$ otherwise. Then

$$
L(f, s)=\sum_{k=0}^{\infty} \frac{1}{(k T+u)^{s}}=\frac{1}{T^{s}} \sum_{k=0}^{\infty} \frac{1}{(k+u / T)^{s}}=\frac{1}{T^{s}} \zeta\left(s, \frac{u}{T}\right)
$$

where $\zeta(s, \alpha)$ is the Hurwitz $\zeta$ function. Therefore the general setting (0.2) encompasses both values of the Hurwitz $\zeta$ function and values of $L$-functions of Dirichlet characters.

As far as we know, Apéry's theorem has never been generalized in this direction; the first natural conjecture in this respect is probably that Catalan's constant $L(\chi, 2)$ is irrational, where $\chi$ is the non-principal character mod 4. The Ball-Rivoal theorem has been generalized to the $L$-function of this character by Rivoal and Zudilin [21]: they have proved (0.3) below with $2+\log 2$ instead of $T+\log 2$, eventhough $T=4$. In the general setting of (0.2), Nishimoto has generalized the Ball-Rivoal theorem as follows [19]:

$$
\operatorname{dim}_{\mathbb{Q}} \operatorname{Span}_{\mathbb{Q}}\{L(f, s), 2 \leq s \leq a, s \equiv p \bmod 2\} \geq \frac{1+o(1)}{T+\log 2} \log a \text { as } a \rightarrow \infty
$$


In the special case where $\sum_{n=1}^{T} f(n) \neq 0$ (which includes the Hurwitz $\zeta$ function but not $L$-functions of non-principal Dirichlet characters), this lower bound appears already in Nash' thesis [17]. The constant $T+\log 2$ in Eq. (0.3) has been refined to $T / 2+\log 2$ in [8], provided $f$ is a Dirichlet character and $T$ is a multiple of 4 . When $f$ is the non-principal character mod 4, this gives as a special case the lower bound of Rivoal and Zudilin [21].

Our first result is that one may replace the constant $T+\log 2$ in Eq. (0.3) with $1+\log 2$, so that the lower bound is uniform in $T$ and is the same as for the Riemann $\zeta$ function.

Theorem 1. Let $T \geq 1$, and $f: \mathbb{Z} \rightarrow \mathbb{C}$ be such that $f(n+T)=f(n)$ for any $n$. Assume that $f$ is not identically zero. Let $p \in\{0,1\}, \varepsilon>0$, and a be sufficiently large (in terms of $T$ and $\varepsilon)$. Then

$$
\operatorname{dim}_{\mathbb{Q}} \operatorname{Span}_{\mathbb{Q}}\{L(f, s), 2 \leq s \leq a, s \equiv p \bmod 2\} \geq \frac{1-\varepsilon}{1+\log 2} \log a .
$$

Of course the same result holds without the restriction $s \equiv p \bmod 2$, but it is weaker and even trivial in some cases where $f$ is even or odd (as noticed above).

In another direction, we generalize the recent result (0.1) to this setting.

Theorem 2. Let $T \geq 1$, and $f: \mathbb{Z} \rightarrow \mathbb{C}$ be such that $f(n+T)=f(n)$ for any $n$. Assume that $f$ is not identically zero. Let $E$ be a finite-dimensional $\mathbb{Q}$-vector space contained in $\mathbb{C}$, $p \in\{0,1\}, \varepsilon>0$, and a be sufficiently large (in terms of $\operatorname{dim} E, T$, and $\varepsilon$ ). Then among the numbers $L(f, s)$ with $2 \leq s \leq a$ and $s \equiv p \bmod 2$, at least

$$
2^{(1-\varepsilon) \frac{\log a}{\log \log a}}
$$

do not belong to $E$.

Taking $E=\mathbb{Q}$ we obtain at least $2^{(1-\varepsilon) \frac{\log a}{\log \log a}}$ irrational values among the numbers $L(f, s)$. The dependence in $a$ is much better than in the lower bound of Theorem 1 ; however we obtain only numbers outside $E$, and not $\mathbb{Q}$-linearly independent numbers.

Before explaining the strategy used in the proofs of Theorems 1 and 2, we would like to state the two main special cases of Theorem 2 explicitly.

Corollary 1. Let $\chi$ be a Dirichlet character; put $p=0$ is $\chi$ is odd, and $p=1$ if $\chi$ is even. Let $E$ be a finite-dimensional $\mathbb{Q}$-vector space contained in $\mathbb{C}$. Let $\varepsilon>0$, and a be sufficiently large (in terms of $\chi, \operatorname{dim} E$, and $\varepsilon$ ). Then among the numbers $L(\chi, s)$ with $2 \leq s \leq a$ and $s \equiv p \bmod 2$, at least $2^{(1-\varepsilon) \frac{\log a}{\log \log a}}$ do not belong to $E$.

Corollary 2. Let $r$ be a positive rational number, and $p \in\{0,1\}$. Let $E$ be a finitedimensional $\mathbb{Q}$-vector space contained in $\mathbb{C}$. Let $\varepsilon>0$, and a be sufficiently large (in terms of $r, \operatorname{dim} E$, and $\varepsilon)$. Then among the numbers

$$
\zeta(s, r)=\sum_{n=0}^{\infty} \frac{1}{(n+r)^{s}}
$$

with $2 \leq s \leq a$ and $s \equiv p \bmod 2$, at least $2^{(1-\varepsilon) \frac{\log a}{\log \log a}}$ do not belong to $E$. 
Corollary 2 is new even for $r=1$, i.e. for the Riemann $\zeta$ function: it is a refinement of (0.1). We would like to emphasize the fact that the proof of [11] does not give this result for $E \neq \mathbb{Q}$ : a different approach is used here, proving linear independence and not only irrationality.

The proof of Theorems 1 and 2 is based on the strategy of [8]: we apply Siegel's linear independence criterion using a general version of Shidlovsky's lemma (namely Theorem 3, stated in $§ 1.3$ and proved in [8] following the approach of Bertrand-Beukers [4] and Bertrand [3]). This makes it necessary to relate the construction to a Padé approximation problem with essentially as many equations as the number of unknowns. In the present paper we adapt this strategy so as to include Sprang's arithmetic lemma [23, Lemma 1.4] and the elimination trick of $[24,23,11]$. The proofs of Theorems 1 and 2 are essentially the same, except for the choice of parameters. It is also possible to prove other results of the same flavour (see Theorem 4 at the end of $\S 3.2$, which implies both Theorem 2 and - up to a multiplicative constant - Theorem 1).

Our construction contains as a special case the one used in [11] to prove (0.1). We prove this in $§ 3.3$; as a byproduct, we relate the construction of [11] to a Padé approximation problem with essentially as many equations as the number of unknowns.

The structure of this paper is as follows. We gather in Proposition 1 the output of the Diophantine construction (see $\S 1.1$ ), and prove it in $\S 1$. Then we deduce Theorems 1 and 2 from Proposition 1 in $§ 3$ using Siegel's linear independence criterion (stated in $§ 2$ ).

\section{Diophantine construction}

In this section we gather the Diophantine part of the proof, namely the construction of linearly independent linear forms. We prove Proposition 1 stated in $\S 1.1$, from which we shall deduce in $\S 3$ the results stated in the introduction. The linear forms are constructed in $\S 1.2$ using series of hypergeometric type. We relate them in $\S 1.4$ to a Padé approximation problem, and then apply a general version of Shidlovsky's lemma (stated in \$1.3). At last, arithmetic and asymptotic properties are dealt with in $\S 1.5$.

\section{$1.1 \quad$ Statement of the result}

Let $a, r, N$ be positive integers such that $1 \leq r<\frac{a}{3 N}$. Let $N \geq 1$, and $f: \mathbb{Z} \rightarrow \mathbb{C}$ be such that $f(m+N)=f(m)$ for any $m$. Assume that $f$ is not identically zero. Let $p \in\{0,1\}$; put

$$
L(f, j)=\sum_{m=1}^{\infty} \frac{f(m)}{m^{j}} \text { for any } j \in\{2, \ldots, a\} .
$$

Let also

$$
\alpha=(4 e)^{(a+1) / N}(2 N)^{2 r+2} r^{-(a+1) / N+4(r+1)} \text { and } \beta=(2 e)^{(a+1) / N}(r+1)^{2 r+2} N^{2 r+2} .
$$


Proposition 1. There exists a constant $c_{1}$, which depends only on a and $N$, with the following property. For any integer multiple $n$ of $N$ there exist integers $s_{k, i}$, with $1 \leq k \leq c_{1}$ and $2 \leq i \leq a+N$, such that:

(i) For any $n$ sufficiently large, the subspace $\mathcal{F}$ of $\mathbb{R}^{a+N-1}$ spanned by the vectors ${ }^{t}\left(s_{k, 2}, \ldots, s_{k, a+N}\right), 1 \leq k \leq c_{1}$, is non-zero and does not depend on $n$.

(ii) For any $k$ and any $i$ we have $\left|s_{k, i}\right| \leq \beta^{n+o(n)}$ as $n \rightarrow \infty$.

(iii) For any $k$ we have, as $n \rightarrow \infty$ :

$$
\left|2(-1)^{p} \sum_{\substack{i=2 \\ i \equiv p \bmod 2}}^{a} s_{k, i} L(f, i)+\sum_{i=0}^{N-1} s_{k, a+1+i} f(i)\right| \leq \alpha^{n+o(n)} .
$$

From now on, the symbols $o(\cdot)$ will be intended as $n \rightarrow \infty$. Since $k \leq c_{1}$, these symbols can be made uniform with respect to $k$.

The integers $s_{k, i}$ depend also implicitly on $n, a, r, N, f$ and $p$. Their values for $i \not \equiv p \bmod 2$ do not appear in the linear combinations of part $(i i i)$, but they could be of interest in other settings. Another feature of this construction is that for $i \leq a$, the integers $s_{k, i}$ depend only on $n, a, r, N$ but not on $f$ or $p$. Probably this could lead to variants of our results in the style of [14] or [7].

Remark 1. In [8] a similar construction is made, where the matrix $\left[s_{k, i}\right]_{i, k}$ has rank a + $N-1$ for $n$ sufficiently large so that the subspace $\mathcal{F}$ of part $(i)$ is equal to $\mathbb{R}^{a+N-1}$. In the present setting we make a different construction to incorporate Sprang's arithmetic lemma (see $§ 1.2$ below), and the matrix $\left[s_{k, i}\right]$ we obtain has rank less than $a+N-1$ for some values of the parameters (see Remark 3 in $\S 1.4$ ): the subspace $\mathcal{F}$ in Proposition 1 is not always equal to $\mathbb{R}^{a+N-1}$.

\subsection{Construction of the linear forms}

In this section we define the numbers $s_{k, i}$ of Proposition 1 (see Eq. (1.17)) and express the linear form of Eq. (1.2) in a more convenient way (see Lemma 1). We postpone until $\S 1.5$ the proof that $s_{k, i} \in \mathbb{Z}$.

As in $\S 1.1$ we let $a, r, N$ be positive integers such that $1 \leq r<\frac{a}{3 N}$. For any integer multiple $n$ of $N$ we let

$$
F(t)=(n / N) !^{(a+1)-(2 r+1) N} \frac{(t-r n)_{(2 r+1) n+1}}{\prod_{h=0}^{n / N}(t+N h)^{a+1}}
$$

where $(\alpha)_{p}=\alpha(\alpha+1) \ldots(\alpha+p-1)$ is the Pochhammer symbol. Note that each factor $t+N h$ of the denominator appears also in the numerator, so that the poles $t=-N h$ of 
this rational function only have order $a$. This rational function $F(t)$ is similar to that of [8], but central factors have been inserted in the numerator to apply Sprang's arithmetic lemma (see Remark 2 below).

In this section we follow the proof of [8], except for Eq. (1.19) which is specific to the function $F$ we consider here. We let

$$
S_{0}(z)=\sum_{t=n+1}^{\infty} F(-t) z^{t} \quad \text { and } \quad S_{\infty}(z)=\sum_{t=1}^{\infty} F(t) z^{-t}
$$

for $z \in \mathbb{C}$ with $|z|=1$; then both series are convergent since the degree $-d_{0}$ of $F$ satisfies

$$
d_{0}:=-\operatorname{deg} F=(a+1)\left(\frac{n}{N}+1\right)-(2 r+1) n-1 \geq 2 .
$$

We let $\omega=e^{2 i \pi / N}$ and for any $\ell \in\{1, \ldots, N\}$ we consider the (inverse) discrete Fourier transform of $f$, defined by

$$
\widehat{f}(\ell)=\frac{1}{N} \sum_{\lambda=1}^{N} f(\lambda) \omega^{-\ell \lambda}
$$

We also let

$$
\delta_{n}=\left(N d_{n / N}\right)^{a+1} N^{(a+1) n / N}, \text { where } d_{j}=\operatorname{lcm}(1,2, \ldots, j) .
$$

The linear forms of Proposition 1 are given by the following lemma. The rational numbers $s_{k, i}$ will be constructed explicitly in the proof (see Eq. (1.17)), and we shall prove in $\S 1.5$ that they are integers.

Lemma 1. For any $1 \leq k \leq d_{0}-1$ there exist rational numbers $s_{k, 2}, \ldots, s_{k, a+N}$ such that

$$
\begin{aligned}
& \delta_{n} \sum_{\ell=1}^{N} \widehat{f}(\ell)\left[\omega^{\ell(k-1)} S_{0}^{(k-1)}\left(\omega^{\ell}\right)+(-1)^{p} \omega^{\ell(1-k)} S_{\infty}^{(k-1)}\left(\omega^{-\ell}\right)\right] \\
& =2(-1)^{p} \sum_{\substack{i=2 \\
i \equiv p \bmod 2}}^{a} s_{k, i} L(f, i)+\sum_{i=0}^{N-1} s_{k, a+1+i} f(i)
\end{aligned}
$$

where $S^{(k-1)}$ is the $(k-1)$-th derivative of a function $S$.

Let us prove Lemma 1. The partial fraction expansion of $F$ reads

$$
F(t)=\sum_{h=0}^{n / N} \sum_{j=1}^{a} \frac{p_{j, h}}{(t+N h)^{j}}
$$

with rational coefficients $p_{j, h}$; we consider

$$
P_{j}(z)=\sum_{h=0}^{n / N} p_{j, h} z^{N h} \in \mathbb{Q}[z]_{\leq n} \text { for any } j \in\{1, \ldots, a\} .
$$


Let $P_{1, j}=P_{j}$ for any $j \in\{1, \ldots, a\}$, and define inductively $P_{k, j} \in \mathbb{Q}(z)$ by

$$
P_{k, j}(z)=P_{k-1, j}^{\prime}(z)-\frac{1}{z} P_{k-1, j+1}(z) \text { for any } k \geq 2 \text { and any } j \in\{1, \ldots, a\},
$$

where $P_{k-1, a+1}=0$ for any $k$. We let also ${ }^{1}$

$$
\begin{gathered}
U_{1}(z)=-\sum_{t=1}^{n} z^{t} \sum_{j=1}^{a} \sum_{h=0}^{\lfloor(t-1) / N\rfloor} \frac{p_{j, h}}{(N h-t)^{j}} \in \mathbb{Q}[z]_{\leq n} \\
\text { and } V_{1}(z)=-\sum_{t=0}^{n-1} z^{t} \sum_{j=1}^{a} \sum_{h=\lceil(t+1) / N\rceil}^{n / N} \frac{p_{j, h}}{(N h-t)^{j}} \in \mathbb{Q}[z]_{\leq n},
\end{gathered}
$$

and define $U_{k}, V_{k}$ for any $k \geq 2$ by the recurrence relations

$$
\begin{gathered}
U_{k}(z)=U_{k-1}^{\prime}(z)-\frac{1}{1-z} P_{k-1,1}(z), \\
V_{k}(z)=V_{k-1}^{\prime}(z)+\frac{1}{z(1-z)} P_{k-1,1}(z) .
\end{gathered}
$$

Then for any $k \geq 1$ we have (as in $[2,10]$ )

$$
\begin{aligned}
& S_{0}^{(k-1)}(z)=U_{k}(z)+\sum_{j=1}^{a} P_{k, j}(z)(-1)^{j} \operatorname{Li}_{j}(z) \\
& \text { and } S_{\infty}^{(k-1)}(z)=V_{k}(z)+\sum_{j=1}^{a} P_{k, j}(z) \operatorname{Li}_{j}(1 / z)
\end{aligned}
$$

Since $P_{j}(z) \in \mathbb{Q}\left[z^{N}\right]$ for any $j \in\{1, \ldots, a\}$, Eq. (1.7) yields $P_{k, j} \in z^{1-k} \mathbb{Q}\left[z^{N}\right]$. This property is very important to us since we shall evaluate $P_{k, j}$ at $N$-th roots of unity. To evaluate in the same way the rational functions $U_{k}, V_{k} \in \mathbb{Q}\left[z, z^{-1}\right]$ for $k \leq d_{0}-1$, we write

$$
z^{k-1} U_{k}(z)=\sum_{\lambda=0}^{N-1} z^{\lambda} U_{k, \lambda}(z) \text { and } z^{k-1} V_{k}(z)=\sum_{\lambda=0}^{N-1} z^{\lambda} V_{k, \lambda}(z)
$$

with $U_{k, \lambda}, V_{k, \lambda} \in \mathbb{Q}\left[z^{N}, z^{-N}\right]$. Then Eqns. (1.12) and (1.13) yield

$$
\begin{aligned}
& z^{k-1} S_{0}^{(k-1)}(z)=\sum_{\lambda=0}^{N-1} z^{\lambda} U_{k, \lambda}(z)+\sum_{j=1}^{a} z^{k-1} P_{k, j}(z)(-1)^{j} \operatorname{Li}_{j}(z) \\
& \text { and } z^{k-1} S_{\infty}^{(k-1)}(z)=\sum_{\lambda=0}^{N-1} z^{\lambda} V_{k, \lambda}(z)+\sum_{j=1}^{a} z^{k-1} P_{k, j}(z) \operatorname{Li}_{j}(1 / z) .
\end{aligned}
$$

\footnotetext{
${ }^{1}$ There is a misprint in the formula that gives $U(z)$ in [8].
} 
We may now define the coefficients $s_{k, i}$ for any $k \geq 1$ by:

$$
\left\{\begin{array}{l}
s_{k, i}=\delta_{n} P_{k, i}(1) \text { for } 2 \leq i \leq a \\
s_{k, a+1+\lambda}=\delta_{n}\left(U_{k, \lambda}(1)+(-1)^{p} V_{k, N-\lambda}(1)\right) \text { for } 0 \leq \lambda \leq N-1,
\end{array}\right.
$$

where $V_{k, N}=V_{k, 0}$; recall that $\delta_{n}=\left(N d_{n / N}\right)^{a+1} N^{(a+1) n / N}$ with $d_{j}=\operatorname{lcm}(1,2, \ldots, j)$. Since $P_{k, j}(z), U_{k, \lambda}(z)$ and $V_{k, N-\lambda}(z)$ are polynomials with rational coefficients, the numbers $s_{k, 2}$, $\ldots, s_{k, a+N}$ are rational. We shall prove in Lemma 3 that they are integers, thanks to the factor $\delta_{n}$. We also point out that $s_{k, i}$ is not defined for $i=1$; actually $P_{k, 1}(1)=0$ for the values of $k$ we are interested in (see (1.18) below).

To conclude the proof of Lemma 1, we shall evaluate Eqns. (1.15) and (1.16) at roots of unity. At the point 1 this is possible since, as in $[8, \S 4.3]$,

for any $k \leq d_{0}-1, \quad P_{k, 1}(1)=0$ and $U_{k}, V_{k}$ do not have a pole at $z=1$.

Now let $k \leq d_{0}-1$, and $z \in \mathbb{C}$ be such that $|z|=1$. Then Eqns. (1.12) to (1.16) hold, upon agreeing that the sums start at $j=2$ if $z=1$; this remark will be used below when $z$ is a $N$-th root of unity.

Let $\Lambda_{k}$ be the right hand side of Eq. (1.6). Using (1.18) the definition (1.17) of $s_{k, i}$ yields

$$
\Lambda_{k}=2 \delta_{n}(-1)^{p} \sum_{\substack{1 \leq j \leq a \\ j \equiv p \bmod 2}} P_{k, j}(1) L(f, j)+\delta_{n} \sum_{\lambda=0}^{N-1}\left(U_{k, \lambda}(1)+(-1)^{p} V_{k, N-\lambda}(1)\right) f(\lambda) .
$$

Now Eq. (1.5) yields

$\sum_{\ell=1}^{N} \widehat{f}(\ell) \omega^{m \ell}=f(m)$ for any $m \in \mathbb{Z}$, so that $\sum_{\ell=1}^{N} \widehat{f}(\ell) \operatorname{Li}_{j}\left(\omega^{\ell}\right)=\sum_{m=1}^{\infty} \frac{f(m)}{m^{j}}=L(f, j)$ for any $j \leq a$.

Therefore we have, since $V_{k, N}=V_{k, 0}$ :

$$
\begin{aligned}
\Lambda_{k}=\quad & \delta_{n} \sum_{j=1}^{a} P_{k, j}(1)\left((-1)^{j}+(-1)^{p}\right) \sum_{\ell=1}^{N} \widehat{f}(\ell) \operatorname{Li}_{j}\left(\omega^{\ell}\right) \\
& +\delta_{n} \sum_{\lambda=0}^{N-1}\left[\left(\sum_{\ell=1}^{N} \widehat{f}(\ell) \omega^{\ell \lambda}\right) U_{k, \lambda}(1)+(-1)^{p}\left(\sum_{\ell=1}^{N} \widehat{f}(\ell) \omega^{-\ell \lambda}\right) V_{k, \lambda}(1)\right] .
\end{aligned}
$$

Then Eqns. (1.15) and (1.16) yield, since $U_{k, \lambda}(z), V_{k, \lambda}(z)$, and $z^{k-1} P_{k, j}(z)$ depend only on $z^{N}$ and $\omega$ is a $N$-th root of unity:

$$
\Lambda_{k}=\delta_{n} \sum_{\ell=1}^{N} \widehat{f}(\ell)\left[\omega^{\ell(k-1)} S_{0}^{(k-1)}\left(\omega^{\ell}\right)+(-1)^{p} \omega^{\ell(1-k)} S_{\infty}^{(k-1)}\left(\omega^{-\ell}\right)\right] .
$$

This concludes the proof of Lemma 1. 
Remark 2. The only difference here with the construction of [8] is that the rational function F has been modified to incorporate Sprang's arithmetic lemma [23, Lemma 1.4]. In our setting this choice of $F$ leads to the following additional property, that will be used in $\S 1.4$ :

$$
U_{1}(z)+V_{1}(z) \in \mathbb{Q}\left[z^{N}\right] .
$$

To prove this property we notice that

$$
U_{1}(z)+V_{1}(z)=-\sum_{t=1}^{n} z^{t} \sum_{j=1}^{a} \sum_{h \neq t / N} \frac{p_{j, h}}{(N h-t)^{j}} ;
$$

for any $t$ which is not a multiple of $N$, the coefficient of $z^{t}$ is $-F(-t)=0$.

\subsection{A general version of Shidlosvky's lemma}

Let $q$ be a positive integer, and $A \in M_{q}(\mathbb{C}(z))$. We fix ${ }^{2} P_{1}, \ldots, P_{q} \in \mathbb{C}[z]$ and $n \in \mathbb{N}=$ $\{0,1,2, \ldots\}$ such that $\operatorname{deg} P_{i} \leq n$ for any $i$. Then with any solution $Y={ }^{t}\left(y_{1}, \ldots, y_{q}\right)$ of the differential system $Y^{\prime}=A Y$ is associated a remainder $R(Y)$ defined by

$$
R(Y)(z)=\sum_{i=1}^{q} P_{i}(z) y_{i}(z) .
$$

Let $\Sigma$ be a finite subset of $\mathbb{C} \cup\{\infty\}$, which may contain singularities of the differential system $Y^{\prime}=A Y$. For each $\sigma \in \Sigma$, let $\left(Y_{j}\right)_{j \in J_{\sigma}}$ be a family of solutions of $Y^{\prime}=A Y$ such that the functions $R\left(Y_{j}\right), j \in J_{\sigma}$, are $\mathbb{C}$-linearly independent and belong to the Nilsson class at $\sigma$ (i.e., have a local expression at $\sigma$ as linear combination of holomorphic functions, with coefficients involving complex powers of $z-\sigma$ and integer powers of $\log (z-\sigma))$. We agree that $J_{\sigma}=\emptyset$ if $\sigma \notin \Sigma$, and define rational functions $P_{k, i} \in \mathbb{C}(z)$ for $k \geq 1$ and $1 \leq i \leq q$ by

$$
\left(\begin{array}{c}
P_{k, 1} \\
\vdots \\
P_{k, q}
\end{array}\right)=\left(\frac{\mathrm{d}}{\mathrm{d} z}+{ }^{t} A\right)^{k-1}\left(\begin{array}{c}
P_{1} \\
\vdots \\
P_{q}
\end{array}\right)
$$

These rational functions $P_{k, i}$ play an important role because they are used to differentiate the remainders (see $[22$, Chapter $3, \S 4]$ ):

$$
R(Y)^{(k-1)}(z)=\sum_{i=1}^{q} P_{k, i}(z) y_{i}(z) .
$$

The following result is proved in [8, Theorem 1.2].

\footnotetext{
${ }^{2}$ We shall check in $\$ 1.4$ that the notation introduced in the present section is consistent with the one used earlier in this paper.
} 
Theorem 3. There exists a positive constant $c_{2}$, which depends only on $A$ and $\Sigma$, with the following property. Assume that, for some $\alpha \in \mathbb{C}$ :

(i) The differential system $Y^{\prime}=A Y$ has a basis of local solutions at $\alpha$ with coordinates in $\mathbb{C}[\log (z-\alpha)]\left[\left[(z-\alpha)^{e}\right]\right]$ for some positive real number $e$.

(ii) We have

$$
\sum_{\sigma \in \Sigma} \sum_{j \in J_{\sigma}} \operatorname{ord}_{\sigma}\left(R\left(Y_{j}\right)\right) \geq(n+1) q-n \# J_{\infty}-\tau
$$

for some $\tau$ with $0 \leq \tau \leq n-c_{2}$.

(iii) All rational functions $P_{k, i}$, with $1 \leq i \leq q$ and $1 \leq k<\tau+c_{2}$, are holomorphic at $z=\alpha$.

Then the matrix $\left[P_{k, i}(\alpha)\right]_{1 \leq i \leq q, 1 \leq k<\tau+c_{2}} \in M_{q, \tau+c_{2}-1}(\mathbb{C})$ has rank at least $q-\# J_{\alpha}$.

In the special case where $\Sigma=\{0\}, \# J_{0}=1, Y_{j}$ is analytic at 0 , and $\alpha \neq 0$ is not a singularity of the differential system $Y^{\prime}=A Y$, this result was used by Shidlovsky to prove the Siegel-Shidlovsky theorem on values of $E$-functions (see [22, Chapter 3, Lemma 10]). The functional part of Shidlovsky's lemma has been generalized by Bertand-Beukers [4] to the case where $\Sigma \subset \mathbb{C}, \# J_{\sigma}=1$ for any $\sigma$, and all functions $Y_{j}$ are obtained by analytic continuation from a single one, analytic at all $\sigma \in \Sigma$. Then Bertrand has allowed [3, Théorème 2] an arbitrary number of solutions at each $\sigma$, assuming that $\infty \notin \Sigma$ and the functions $Y_{j}, j \in J_{\sigma}$, are analytic at $\sigma$. The proof [8] of Theorem 3 follows the approach of Bertand-Beukers and Bertrand, based on differential Galois theory.

An important feature of Theorem 3 is that $\alpha$ may be a singularity of the differential system $Y^{\prime}=A Y$, and/or an element of $\Sigma$. Both happen in the present paper, where $\alpha=1$ (see $\S 1.4$ where Theorem 3 is applied to prove Lemma 2). If $\alpha \notin \Sigma$ then $J_{\alpha}=\emptyset$ so that Theorem 3 yields a matrix of maximal rank $q$. On the other hand, if $\alpha \in \Sigma$ then the $\# J_{\alpha}$ linearly independent linear combinations of the rows of the matrix $\left[P_{k, i}(z)\right]_{i, k}$ corresponding to $R\left(Y_{j}\right), j \in J_{\alpha}$, may vanish at $\alpha$ : the lower bound $q-\# J_{\alpha}$ is best possible. In the setting of $\S 1.4$ we have $\alpha=1$ and $J_{1}=\{1\}$ so that Theorem 3 yields $\operatorname{rk}\left[P_{k, i}(1)\right] \geq q-1$. Now (1.18) in the proof of Lemma 1 shows that $P_{k, 1}(1)=0$ for any $k<\tau+c_{2}$ (since $\tau+c_{2} \leq d_{0}$ because $\tau$ and $c_{2}$ are independent from $n$ whereas $d_{0}$ tends to $\infty$ with $\left.n\right)$. Therefore the matrix $\left[P_{k, i}(1)\right]_{1 \leq i \leq q, 1 \leq k<\tau+c_{2}}$ has rank equal to $q-1$. Removing the first row, which is identically zero, yields a matrix of rank $q-1$ equal to the number of rows.

\subsection{Padé approximation and application of Shidlovsky's lemma}

In this section we prove part $(i)$ of Proposition 1 for the numbers $s_{k, i}$ constructed in $\S 1.2$.

Lemma 2. Let $s_{k, i}$ be defined by Eq. (1.17). Then there exists a positive constant $c_{1}$ (which depends only on a and $N$ ) such that for any $n$ sufficiently large, the subspace $\mathcal{F}$ of $\mathbb{R}^{a+N-1}$ spanned by the vectors ${ }^{t}\left(s_{k, 2}, \ldots, s_{k, a+N}\right), 1 \leq k \leq c_{1}$, is non-zero and does not depend on $n$. 
The proof of Lemma 2 falls into 3 steps. To begin with, we construct a Padé approximation problem related to our construction, with essentially as many equations as the number of unknowns; notice that this problem is not the same as in [8], since the function $F$ used in the construction is different. Then we apply a general version of Shidlovsky's lemma, namely Theorem 3 stated in $\S 1.3$. This provides a matrix $P$ with linearly independent rows. At last, we relate the numbers $s_{k, i}$ to $P$ by constructing a matrix $M$ such that $\left[s_{k, i}\right]_{i, k}=M P$. The point is that $M$ does not depend on $n$ (whereas $P$ and $\left[s_{k, i}\right]$ do). The subspace spanned by the columns of $\left[s_{k, i}\right]_{i, k}$ is the same as the one spanned by the columns of $M$ : it does not depend on $n$.

Step 1: Construction of the Padé approximation problem.

We recall from $\S 1.2$ that

$$
\begin{gathered}
F(t)=(n / N) !^{(a+1)-(2 r+1) N} \frac{(t-r n)_{(2 r+1) n+1}}{\prod_{h=0}^{n / N}(t+N h)^{a+1}}, \\
S_{0}(z)=\sum_{t=n+1}^{\infty} F(-t) z^{t}=U_{1}(z)+\sum_{j=1}^{a} P_{j}(z)(-1)^{j} \operatorname{Li}_{j}(z), \\
\text { and } S_{\infty}(z)=\sum_{t=1}^{\infty} F(t) z^{-t}=V_{1}(z)+\sum_{j=1}^{a} P_{j}(z) \operatorname{Li}_{j}(1 / z) .
\end{gathered}
$$

Since $P_{j}(z) \in \mathbb{C}\left[z^{N}\right]$ for any $j \in\{1, \ldots, a\}$, we have $P_{j}\left(\omega^{\ell} z\right)=P_{j}(z)$ for any $\ell \in \mathbb{Z}$. Therefore letting

$$
R_{0, \ell}(z)=S_{0}\left(\omega^{\ell} z\right), \quad R_{\infty, \ell}(z)=S_{\infty}\left(\omega^{\ell} z\right), \quad \bar{P}_{0, \ell}(z)=U_{1}\left(\omega^{\ell} z\right), \quad \bar{P}_{\infty, \ell}(z)=V_{1}\left(\omega^{\ell} z\right)
$$

for any $\ell \in\{1, \ldots, N\}$, we have

$$
\begin{aligned}
& R_{0, \ell}(z)=\bar{P}_{0, \ell}(z)+\sum_{j=1}^{a} P_{j}(z)(-1)^{j} \operatorname{Li}_{j}\left(\omega^{\ell} z\right)=O\left(z^{(r+1) n+1}\right), \quad z \rightarrow 0, \\
& \text { and } R_{\infty, \ell}(z)=\bar{P}_{\infty, \ell}(z)+\sum_{j=1}^{a} P_{j}(z) \operatorname{Li}_{j}\left(\frac{1}{\omega^{\ell} z}\right)=O\left(z^{-r n-1}\right), \quad z \rightarrow \infty .
\end{aligned}
$$

Moreover, recall that $d_{0}=-\operatorname{deg} F=(a+1)\left(\frac{n}{N}+1\right)-(2 r+1) n-1$; Lemma 3 of [10] shows that

$$
\sum_{j=1}^{a} P_{j}(z)(-1)^{j-1} \frac{(\log z)^{j-1}}{(j-1) !}=O\left((z-1)^{d_{0}-1}\right), \quad z \rightarrow 1 .
$$

Using again the fact that $P_{j}\left(\omega^{-\ell} z\right)=P_{j}(z)$, we obtain for any $\ell \in\{1, \ldots, N\}$ :

$$
R_{\omega^{\ell}}(z):=\sum_{j=1}^{a} P_{j}(z)(-1)^{j-1} \frac{\left(\log \left(\omega^{-\ell} z\right)\right)^{j-1}}{(j-1) !}=O\left(\left(z-\omega^{\ell}\right)^{d_{0}-1}\right), \quad z \rightarrow \omega^{\ell} .
$$


The new point here, with respect to [8], is that Eq. (1.19) in Remark 2 shows that $\bar{P}=\bar{P}_{0, \ell}+\bar{P}_{\infty, \ell}$ does not depend on $\ell$. Therefore Eq. (1.23) can be written as

$$
R_{0, \ell}(z)=\bar{P}(z)-\bar{P}_{\infty, \ell}(z)+\sum_{j=1}^{a} P_{j}(z)(-1)^{j} \operatorname{Li}_{j}\left(\omega^{\ell} z\right)=O\left(z^{(r+1) n+1}\right), \quad z \rightarrow 0
$$

We have obtained a Padé approximation problem with $(a+N+1)(n+1)$ unknowns, namely the coefficients of $\bar{P}(z), P_{j}(z)$ for $1 \leq j \leq a$, and $\bar{P}_{\infty, \ell}$ for $1 \leq \ell \leq N$. Eqns. (1.24), (1.25) and (1.26) amount to

$$
N((r+1) n+1)+N\left(d_{0}-1\right)+N((r+1) n+1)=(a+N+1)(n+1)-\tau
$$

linear equations, where $\tau=a+1-a N$ is the difference between the number of unknowns and the number of equations. If $N=1$ then $\tau=1$ : this is exactly the Padé approximation problem of [10, Theorem 1], which has a unique solution up to proportionality. However if $N \geq 2$ then $\tau<0$ : we have solved a linear system with (slightly) more equations than the number of unknowns.

Step 2: Application of Shidlovsky's lemma.

Let us introduce some notation to fit into the context of $\S 1.3$, and check the assumptions of Theorem 3. Let $q=a+N+1$, and $A \in M_{q}(\mathbb{C}(z))$ be the matrix of which the coefficients $A_{i, j}$ are given by:

$$
\left\{\begin{array}{l}
A_{i, i-1}(z)=\frac{-1}{z} \text { for any } i \in\{2, \ldots, a\} \\
A_{1, a+1}(z)=\frac{1}{z} \\
A_{1, a+1+\ell}(z)=\frac{1}{z\left(1-\omega^{\ell} z\right)} \text { for any } \ell \in\{1, \ldots, N\}
\end{array}\right.
$$

and all other coefficients are zero. We consider the following solutions of the differential system $Y^{\prime}=A Y$, with $1 \leq \ell \leq N$ :

$$
\begin{gathered}
Y_{0, \ell}(z)={ }^{t}\left(-\operatorname{Li}_{1}\left(\omega^{\ell} z\right), \operatorname{Li}_{2}\left(\omega^{\ell} z\right), \ldots,(-1)^{a} \operatorname{Li}_{a}\left(\omega^{\ell} z\right), 1,0, \ldots, 0,-1,0, \ldots, 0\right), \\
Y_{\infty, \ell}(z)={ }^{t}\left(\operatorname{Li}_{1}\left(\frac{1}{\omega^{\ell} z}\right), \operatorname{Li}_{2}\left(\frac{1}{\omega^{\ell} z}\right), \ldots, \operatorname{Li}_{a}\left(\frac{1}{\omega^{\ell} z}\right), 0,0, \ldots, 0,1,0, \ldots, 0\right), \\
Y_{\omega^{\ell}}(z)={ }^{t}\left(1,-\log \left(\omega^{-\ell} z\right), \frac{\left(\log \left(\omega^{-\ell} z\right)\right)^{2}}{2 !}, \ldots,(-1)^{a-1} \frac{\left(\log \left(\omega^{-\ell} z\right)\right)^{a-1}}{(a-1) !}, 0,0, \ldots, 0\right)
\end{gathered}
$$

where the coefficient -1 in $Y_{0, \ell}(z)$ (resp. 1 in $\left.Y_{\infty, \ell}(z)\right)$ is in position $a+1+\ell$.

We let $J_{0}=\{(0,1),(0,2), \ldots,(0, N)\}, J_{\infty}=\{(\infty, 1),(\infty, 2), \ldots,(\infty, N)\}, J_{\omega^{\ell}}=\left\{\omega^{\ell}\right\}$ for $1 \leq \ell \leq N$, and $\Sigma=\{0, \infty\} \cup\left\{\omega^{\ell}, 1 \leq \ell \leq N\right\}$, so that we have a solution $Y_{j}$ for each $j \in J_{\sigma}, \sigma \in \Sigma$.

We also let $P_{a+1}(z)=\bar{P}(z)$ (which is equal to $\bar{P}_{0, \ell}(z)+\bar{P}_{\infty, \ell}(z)=\left(U_{1}+V_{1}\right)\left(\omega^{\ell} z\right)$ for any $\ell$ ), and $P_{a+1+\ell}(z)=\bar{P}_{\infty, \ell}(z)=V_{1}\left(\omega^{\ell} z\right)$ for any $\ell \in\{1, \ldots, N\}$. Then we have 
polynomials $P_{1}(z), \ldots, P_{q}(z)$ of degree at most $n$, and with the notation of $\S 1.3$ the remainders associated with the local solutions $Y_{j}, j \in J_{\sigma}, \sigma \in \Sigma$, are exactly the functions that appear in the Padé approximation problem of Step 1: $R\left(Y_{0, \ell}\right)=R_{0, \ell}(z), R\left(Y_{\infty, \ell}\right)=$ $R_{\infty, \ell}(z)$, and $R\left(Y_{\omega^{\ell}}\right)=R_{\omega^{\ell}}(z)$ for any $\ell \in\{1, \ldots, N\}$.

Since $P_{a}$ is not the zero polynomial, we have $R_{\omega^{\ell}}(z) \neq 0$ for any $\ell$; the functions $R_{0,1}(z)$, $\ldots, R_{0, N}(z)$ (resp. $\left.R_{\infty, 1}(z), \ldots, R_{\infty, N}(z)\right)$ are $\mathbb{C}$-linearly independent (see [8, Lemma 2$]$ ).

Eqns. (1.24), (1.25) and (1.26) yield $\operatorname{ord}_{\infty}\left(R_{\infty, \ell}(z)\right) \geq r n+1, \operatorname{ord}_{\omega^{\ell}}\left(R_{\omega^{\ell}}(z)\right) \geq d_{0}-1$ and $\operatorname{ord}_{0}\left(R_{0, \ell}(z)\right) \geq(r+1) n+1$ for any $\ell \in\{1, \ldots, N\}$, so that

$\sum_{\sigma \in \Sigma} \sum_{j \in J_{\sigma}} \operatorname{ord}_{\sigma} R_{j}(z) \geq(2 r+1) N n+N\left(d_{0}+1\right)=(n+1) q-n N-\tau$ with $\tau=a+1-a N ;$ here $q=a+N+1$, and we recall that $d_{0}=-\operatorname{deg} F=(a+1)\left(\frac{n}{N}+1\right)-(2 r+1) n-1$. As above, $\tau$ is exactly the difference between the number of unknowns and the number of equations in the Padé approximation problem of Step 1.

The definition (1.20) of $P_{k, i}$ is consistent with the one given (for $i \leq a$ ) by Eq. (1.7) in §1.2. We have $\tau=a+1-a N$, so that for $n$ sufficiently large $\tau+c_{2} \leq d_{0}$ where $c_{2}$ is the constant given by Theorem 3. Therefore (1.18) shows that $U_{k}$ and $V_{k}$ are holomorphic at $z=1$ for any $k<\tau+c_{2}$. Eqns. (1.7), (1.10) and (1.11) imply that they are holomorphic at all other roots of unity. Now Eqns. (1.20), (1.10) and (1.11) yield

$$
P_{k, a+1}(z)=\omega^{\ell(k-1)}\left(U_{k}\left(\omega^{\ell} z\right)+V_{k}\left(\omega^{\ell} z\right)\right) \text { and } P_{k, a+1+\ell}(z)=\omega^{\ell(k-1)} V_{k}\left(\omega^{\ell} z\right)
$$

for any $\ell \in\{1, \ldots, N\}$, by induction on $k \geq 1$. Therefore all $P_{k, i}$, with $k<\tau+c_{2}$ and $1 \leq i \leq q$, are holomorphic at 1 .

We have checked all assumptions of Theorem 3 for $n$ sufficiently large: the matrix $\left[P_{k, i}(1)\right]_{1 \leq i \leq q, 1 \leq k<\tau+c_{2}}$ has rank at least $q-\# J_{1}=q-1$. Now (1.18) implies $P_{k, 1}(1)=0$ for any $k<\tau+c_{2}$, so that we may remove the first row: the matrix $P=\left[P_{k, i}(1)\right]_{2 \leq i \leq q, 1 \leq k<\tau+c_{2}}$ has rank $q-1$, equal to its number of rows.

Step 3: Expression of $s_{k, i}$ in terms of $P$ and conclusion.

We shall now compute a matrix $M$ independent from $n$ such that $\left[s_{k, i}\right]_{i, k}=M P$; recall that the coefficients $s_{k, i}$ and the matrix $P$ depend on $n$.

To begin with, Eq. (1.14) with $z=\omega^{\ell}$ yields

$$
\omega^{(k-1) \ell} U_{k}\left(\omega^{\ell}\right)=\sum_{\lambda=0}^{N-1} \omega^{\lambda \ell} U_{k, \lambda}(1) \text { for any } \ell \in \mathbb{Z},
$$

since $U_{k, \lambda}(z) \in \mathbb{Q}\left[z^{N}, z^{-N}\right]$. Therefore we have

$$
U_{k, \lambda}(1)=\frac{1}{N} \sum_{\ell=1}^{N} \omega^{(k-1-\lambda) \ell} U_{k}\left(\omega^{\ell}\right) \text { for any } 0 \leq \lambda \leq N-1,
$$


and the same relation holds with $V_{k, \lambda}$ and $V_{k}$. Using Eq. (1.27) we deduce that

$$
V_{k, \lambda}(1)=\frac{1}{N} \sum_{\ell=1}^{N} \omega^{-\lambda \ell} P_{k, a+1+\ell}(1) \text { for } 0 \leq \lambda \leq N-1,
$$

and also for $\lambda=N$ since $V_{k, N}=V_{k, 0}$, and

$$
U_{k, \lambda}(1)=\left\{\begin{array}{l}
-\frac{1}{N} \sum_{\ell=1}^{N} \omega^{-\lambda \ell} P_{k, a+1+\ell}(1) \text { if } 1 \leq \lambda \leq N-1, \\
P_{k, a+1}(1)-\frac{1}{N} \sum_{\ell=1}^{N} P_{k, a+1+\ell}(1) \text { if } \lambda=0 .
\end{array}\right.
$$

Therefore the definition (1.17) of $s_{k, i}$ can be translated as

$$
s_{k, i}=\sum_{j=2}^{q} m_{i, j} P_{k, j}(1)
$$

for any $2 \leq i \leq a+N$ and any $1 \leq k \leq d_{0}-1$, where the coefficients $m_{i, j}$ are defined for $2 \leq i \leq a+N$ and $2 \leq j \leq q=a+N+1$ by

$$
\left\{\begin{array}{l}
m_{i, i}=\delta_{n} \text { for } 2 \leq i \leq a+1 \\
m_{a+1, a+1+\ell}=\frac{\delta_{n}}{N}\left((-1)^{p}-1\right) \text { for } 1 \leq \ell \leq N \\
m_{a+1+\lambda, a+1+\ell}=\frac{\delta_{n}}{N}\left((-1)^{p} \omega^{\lambda \ell}-\omega^{-\lambda \ell}\right) \text { for } 1 \leq \ell \leq N \text { and } 1 \leq \lambda \leq N-1 \\
m_{i, j}=0 \text { for all other pairs }(i, j) .
\end{array}\right.
$$

Let us choose now the constant $c_{1}$ of Lemma 2; the same constant appears in Proposition 1. We take $c_{1}=\tau+c_{2}-1$; this constant depends only on $a$ and $N$. We consider the matrices $M=\left[m_{i, j}\right]_{2 \leq i \leq a+N, 2 \leq j \leq q}$ and $P=\left[P_{k, j}(1)\right]_{2 \leq j \leq q, 1 \leq k \leq c_{1}}$. Then Eq. (1.29) means that

$$
\left[s_{k, i}\right]_{2 \leq i \leq a+N, 1 \leq k \leq c_{1}}=M P .
$$

Both $M$ and $P$ have coefficients in $\mathbb{Q}(\omega)$; recall that the coefficients $s_{k, i}$ of $M P$ are rational numbers, and we shall prove in $\S 1.5$ that they are integers. Let $\mathcal{F}$ denote the subspace of $\mathbb{R}^{a+N-1}$ spanned by the $q-1$ columns ${ }^{t}\left(m_{2, j}, \ldots, m_{a+N, j}\right)$ of $M$. Now assume that $n$ is sufficiently large; then we have proved in Step 2 that the $q-1$ rows of $P$ are linearly independent. Therefore Eq. (1.31) shows that $\mathcal{F}$ is equal to the subspace spanned by columns ${ }^{t}\left(s_{k, 2}, \ldots, s_{k, a+N}\right)$ of the matrix $\left[s_{k, i}\right]_{i, k}$. Since $M$ does not depend on $n$, neither does $\mathcal{F}$ : this concludes the proof of Lemma 2 .

Remark 3. Let us prove that in Lemma 2, the subspace $\mathcal{F}$ is not always equal to $\mathbb{R}^{a+N-1}$ (i.e., that the matrix $\left[s_{k, i}\right]$ may have rank less than its number of rows, namely $a+N-1$ ). Consider the case where $p$ and $N$ are even (so that $\omega^{N / 2}=-1$ ). Then the definition (1.30) of the matrix $M$ in Step 3 above yields $m_{a+1+N / 2, j}=0$ for any $j$, so that Eq. (1.29) implies $s_{k, a+1+N / 2}=0$ for any $k$ : the matrix $\left[s_{k, i}\right]_{i, k}$ has a zero row. This phenomenon does not occur in [8]; it comes from the new property (1.19) obtained in Remark 2. Indeed a direct 
proof that $s_{k, a+1+N / 2}=0$ can be obtained as follows, using Eqns. (1.17), (1.28), and (1.27) but not the matrix $M$ :

$$
\begin{aligned}
s_{k, a+1+N / 2} & =\delta_{n}\left(U_{k, N / 2}(1)+V_{k, N / 2}(1)\right) \\
& =\frac{\delta_{n}}{N} \sum_{\ell=1}^{N} \omega^{(k-1-N / 2) \ell}\left(U_{k}+V_{k}\right)\left(\omega^{\ell}\right)=\frac{\delta_{n}}{N} P_{k, a+1}(1) \sum_{\ell=1}^{N}(-1)^{\ell}=0 .
\end{aligned}
$$

\subsection{Arithmetic and Asymptotic Properties}

In this section we conclude the proof of Proposition 1 stated in $\S 1.1$, by proving parts $(i i)$ and $(i i i)$ and the fact that the $s_{k, i}$ are integers. Recall that

$$
\alpha=(4 e)^{(a+1) / N}(2 N)^{2 r+2} r^{-(a+1) / N+4(r+1)} \text { and } \beta=(2 e)^{(a+1) / N}(r+1)^{2 r+2} N^{2 r+2} .
$$

Lemma 3. We have $s_{k, i} \in \mathbb{Z}$ for any $i \in\{2, \ldots, a+N\}$ and any $k \leq d_{0}-1$, and as $n \rightarrow \infty$ :

$$
\left|2(-1)^{p} \sum_{\substack{i=2 \\ i \equiv p \bmod 2}}^{a} s_{k, i} L(f, i)+\sum_{i=0}^{N-1} s_{k, a+1+i} f(i)\right| \leq \alpha^{n+o(n)} \text { and } \max _{2 \leq i \leq a+N}\left|s_{k, i}\right| \leq \beta^{n(1+o(1))} .
$$

In this lemma and throughout this section, we denote by $o(1)$ any sequence that tends to 0 as $n \rightarrow \infty$; it usually depends also on $a, r, N$, and $k$ (but the dependence in $k$ is not significant since $k$ is bounded by $d_{0}-1$, which depends only on $\left.n, a, r, N\right)$. We also recall that $d_{n}$ is the least common multiple of $1,2, \ldots, n$.

We shall prove two lemmas now; the deduction of Lemma 3 from these lemmas (using Lemma 1 proved in $\S 1.2)$ is exactly the same as the proof of Proposition 1 in [8, §4.5]. Recall from $\S 1.2$ that

$$
F(t)=(n / N) !^{(a+1)-(2 r+1) N} \frac{(t-r n)_{(2 r+1) n+1}}{\prod_{h=0}^{n / N}(t+N h)^{a+1}}=\sum_{h=0}^{n / N} \sum_{j=1}^{a} \frac{p_{j, h}}{(t+N h)^{j}} .
$$

Lemma 4. For any $j \in\{1, \ldots, a\}$ and any $h \in\{0, \ldots, n / N\}$ we have

$$
\begin{gathered}
\left(N d_{n / N}\right)^{a+1-j} N^{(a+1) n / N} p_{j, h} \in \mathbb{Z} \\
\text { and }\left|p_{j, h}\right| \leq\left(2^{(a+1) / N} N^{2(r+1)-(a+1) / N}(r+1)^{2 r+2}\right)^{n(1+o(1))}
\end{gathered}
$$

where $o(1)$ is a sequence that tends to 0 as $n \rightarrow \infty$ and may depend also on $N$, a, and $r$. 
Proof of Lemma 4: We follow the approach of [5] by letting

$$
\begin{aligned}
& F_{0}(t)=\frac{(n / N) !}{\prod_{h=0}^{n / N}(t+N h)}=\sum_{h=0}^{n / N} \frac{(-1)^{h} N^{-n / N}\left(\begin{array}{c}
n / N \\
h
\end{array}\right)}{t+N h}, \\
& G_{i}(t)=\frac{(t-i n / N)_{n / N}}{\prod_{h=0}^{n / N}(t+N h)}=\sum_{h=0}^{n / N} \frac{(-1)^{h+n / N} N^{-n / N}\left(\begin{array}{c}
n / N \\
h
\end{array}\right)\left(\begin{array}{c}
N h+i n / N \\
n / N
\end{array}\right)}{t+N h} \text { for } 1 \leq i \leq r N, \\
& H_{i}(t)=\frac{(t+1+i n / N)_{n / N}}{\prod_{h=0}^{n / N}(t+N h)}=\sum_{h=0}^{n / N} \frac{(-1)^{h} N^{-n / N}\left(\begin{array}{c}
n / N \\
h
\end{array}\right)\left(\begin{array}{c}
-N h+(i+1) n / N \\
n / N
\end{array}\right)}{t+N h} \text { for } 0 \leq i \leq(r+1) N-1 .
\end{aligned}
$$

Then the partial fraction expansion of

$$
F(t)=F_{0}(t)^{a+1-(2 r+1) N} t G_{1}(t) \ldots G_{r N}(t) H_{0}(t) \ldots H_{(r+1) N-1}(t)
$$

can be obtained by multiplying those of $F_{0}, G_{i}$ and $H_{i}$ using repeatedly the formulas $\frac{t}{t+N h}=1-\frac{N h}{t+N h}$ and

$$
\frac{1}{(t+N h)\left(t+N h^{\prime}\right)^{\ell}}=\frac{1}{N^{\ell}\left(h^{\prime}-h\right)^{\ell}(t+N h)}-\sum_{i=1}^{\ell} \frac{1}{N^{\ell+1-i}\left(h^{\prime}-h\right)^{\ell+1-i}\left(t+N h^{\prime}\right)^{i}}
$$

with $h \neq h^{\prime}$. The denominator of $p_{j, h}$ comes both from this formula (and this contribution divides $\left.\left(N d_{n / N}\right)^{a+1-j}\right)$ and from the denominators of the coefficients in the partial fraction expansions of $F_{0}, G_{i}, H_{i}$ (which belong to $N^{-n / N} \mathbb{Z}$, so that $N^{(a+1) n / N}$ accounts for this contribution). This concludes the proof of (1.32).

On the other hand, bounding from above the coefficients of the partial fraction expansions of $F_{0}, G_{i}, H_{i}$ yields

$$
\left|p_{j, h}\right| \leq n^{O(1)} N^{-(a+1) n / N} 2^{(a+1) n / N} \prod_{i=1}^{r N} \frac{(n+i n / N) !}{(n / N) !(n+(i-1) n / N) !} \prod_{i=0}^{(r+1) N-1} \frac{((i+1) n / N) !}{(n / N) !(i n / N) !}
$$

where $O(1)$ is a constant depending only on $a, r, N$ which can be made explicit (see [5] for details). Simplifying the products and using the bound $\frac{m !}{m_{1} ! \ldots m_{c} !} \leq c^{m}$ valid when $m_{1}+\ldots+m_{c}=m$, one obtains

$$
\left|p_{j, h}\right| \leq n^{O(1)}(2 / N)^{(a+1) n / N} \frac{((r+1) n) !^{2}}{n !(n / N) !(2 r+1) N} \leq n^{O(1)}(2 / N)^{(a+1) n / N}((r+1) N)^{2(r+1) n} .
$$

This concludes the proof of Lemma 4.

The proof of the following lemma is inspired by that of [23, Lemma 1.4]. Recall that $U_{1}$ and $V_{1}$ are defined in Eqns. (1.8) and (1.9), and that

$$
\delta_{n}=\left(N d_{n / N}\right)^{a+1} N^{(a+1) n / N} .
$$


Lemma 5. The polynomials $\delta_{n} U_{1}(z)$ and $\delta_{n} V_{1}(z)$ have integer coefficients.

Proof of Lemma 5: Recall from Eq. (1.8) that

$$
U_{1}(z)=-\sum_{t=1}^{n} z^{t} \sum_{j=1}^{a} \sum_{h=0}^{\lfloor(t-1) / N\rfloor} \frac{p_{j, h}}{(N h-t)^{j}} .
$$

Assume that $\delta_{n} U_{1}(z)$ does not have integer coefficients. Then there exists $t \in\{1, \ldots, n\}$ such that

$$
\sigma:=\sum_{j=1}^{a} \sum_{h=0}^{\lfloor(t-1) / N\rfloor} \frac{\left(N d_{n / N}\right)^{a+1} N^{(a+1) n / N} p_{j, h}}{(N h-t)^{j}} \notin \mathbb{Z} .
$$

Let $p_{j, h}^{\prime}=\left(N d_{n / N}\right)^{a+1-j} N^{(a+1) n / N} p_{j, h}$, which is an integer thanks to Lemma 4. Then we have

$$
\sigma=\sum_{j=1}^{a} \sum_{h=0}^{\lfloor(t-1) / N\rfloor} \frac{d_{n / N}^{j} p_{j, h}^{\prime}}{(h-t / N)^{j}} .
$$

If $N$ divides $t$ then $\frac{t}{N}-h$ is a positive integer less than or equal to $n / N$, so that it divides $d_{n / N}$ : this contradicts the assumption $\sigma \notin \mathbb{Z}$. Therefore $N$ does not divide $t$, so that $F(-t)=0$.

Since $\sigma \notin \mathbb{Z}$ there exists $h_{0}$ such that

$$
\sum_{j=1}^{a} \frac{d_{n / N}^{j} p_{j, h_{0}}^{\prime}}{\left(h_{0}-t / N\right)^{j}} \notin \mathbb{Z}
$$

Now $F(-t)=0$ so that

$$
\sum_{j=1}^{a} \sum_{\substack{h=0 \\ h \neq h_{0}}}^{n / N} \frac{d_{n / N}^{j} p_{j, h}^{\prime}}{(h-t / N)^{j}}=-\sum_{j=1}^{a} \frac{d_{n / N}^{j} p_{j, h_{0}}^{\prime}}{\left(h_{0}-t / N\right)^{j}} \notin \mathbb{Z} .
$$

This rational number has negative $p$-adic valuation for some prime number $p$. Therefore there exist $h_{1} \neq h_{0}$ and $j_{0}, j_{1}$ such that

$$
v_{p}\left(\frac{d_{n / N}^{j_{1}}}{\left(h_{1}-t / N\right)^{j_{1}}}\right)<0 \text { and } v_{p}\left(\frac{d_{n / N}^{j_{0}}}{\left(h_{0}-t / N\right)^{j_{0}}}\right)<0 .
$$

This implies

$$
\min \left(v_{p}\left(h_{1}-t / N\right), v_{p}\left(h_{0}-t / N\right)\right)>v_{p}\left(d_{n / N}\right)
$$

so that $v_{p}\left(h_{0}-h_{1}\right)>v_{p}\left(d_{n / N}\right)$. This is a contradiction since $1 \leq\left|h_{0}-h_{1}\right| \leq n / N$. This concludes the proof that $\delta_{n} U_{1}(z) \in \mathbb{Z}[z]$; the same proof works for $\delta_{n} V_{1}(z)$. 


\section{Siegel's linear independence criterion}

The following criterion is based on Siegel's ideas (see for instance [6, p. 81-82 and 215-216], $[16, \S 3]$ or $[15$, Proposition 4.1]).

Proposition 2. Let $\theta_{1}, \ldots, \theta_{q}$ be complex numbers, not all zero. Let $\tau>0$, and $\left(Q_{n}\right)$ be a sequence of real numbers with limit $+\infty$. Let $\mathcal{N}$ be an infinite subset of $\mathbb{N}, K \geq 1$, and for any $n \in \mathcal{N}$ let $L^{(n)}=\left[\ell_{k, i}^{(n)}\right]_{1 \leq i \leq q, 1 \leq k \leq K}$ be a matrix with integer coefficients such that as $n \rightarrow \infty$ with $n \in \mathcal{N}$ :

$$
\begin{gathered}
\max _{i, k}\left|\ell_{k, i}^{(n)}\right| \leq Q_{n}^{1+o(1)} \\
\text { and } \max _{1 \leq k \leq K}\left|\ell_{k, 1}^{(n)} \theta_{1}+\ldots+\ell_{k, q}^{(n)} \theta_{q}\right| \leq Q_{n}^{-\tau+o(1)} .
\end{gathered}
$$

Assume also that the subspace $\mathcal{F}$ of $\mathbb{R}^{q}$ spanned by the columns ${ }^{t}\left(\ell_{k, 1}^{(n)}, \ldots, \ell_{k, q}^{(n)}\right)$ of $L^{(n)}$ is non-zero and independent from $n \in \mathcal{N}$ (provided $n$ is large enough). Then we have

$$
\operatorname{dim}_{\mathbb{Q}} \operatorname{Span}_{\mathbb{Q}}\left(\theta_{1}, \ldots, \theta_{q}\right) \geq \tau+1 .
$$

The usual version of this criterion (see for instance [9, Theorem 4]) is the same statement, but the assumption on $\mathcal{F}$ is replaced by the assumption that $L^{(n)}$ is invertible. The latter is stronger, since it is equivalent to asking $\mathcal{F}=\mathbb{R}^{q}$ for any $n$. Indeed if $\mathcal{F}=\mathbb{R}^{q}$ then $L^{(n)}$ has rank $q$ : for each $n$ we may extract $q$ linearly independent columns of $L^{(n)}$, and obtain an invertible matrix to which [9, Theorem 4] applies. The point is that we shall apply Proposition 2 to the matrices $\left[s_{k, i}\right]$ constructed in Proposition 1, and the subspace $\mathcal{F}$ is not always equal to $\mathbb{R}^{q}$ (see Remark 3 in $\S 1.4$ ).

Let us prove Proposition 2 now. Denote by $\mathcal{F}$ the image of $L^{(n)}$, assumed to be independent from $n \in \mathcal{N}$ (provided $n$ is large enough). Let $p=\operatorname{dim} \mathcal{F}$. Permuting $\theta_{1}, \ldots, \theta_{q}$ if necessary, we may assume that a system of linear equations of $\mathcal{F}$ is given by

$$
x_{t}=\sum_{i=1}^{p} \mu_{t, i} x_{i} \text { for } p+1 \leq t \leq q, \text { with } \mu_{t, i} \in \mathbb{Q} .
$$

We point out that the coefficients $\mu_{t, i}$ are rational numbers because the matrices $L^{(n)}$ have integer coefficients. Since ${ }^{t}\left(\ell_{k, 1}^{(n)}, \ldots, \ell_{k, q}^{(n)}\right) \in \mathcal{F}$ for any $1 \leq k \leq K$ and any $n \in \mathcal{N}$ sufficiently large, Eq. (2.1) yields

$$
\sum_{i=1}^{p} \ell_{k, i}^{(n)} \theta_{i}=\sum_{i=1}^{p} \ell_{k, i}^{(n)}\left(\theta_{i}+\sum_{t=p+1}^{q} \mu_{t, i} \theta_{t}\right)=\sum_{i=1}^{p} \ell_{k, i}^{(n)} \theta_{i}^{\prime}
$$

upon letting $\theta_{i}^{\prime}=\theta_{i}+\sum_{t=p+1}^{q} \mu_{t, i} \theta_{t}$ for $1 \leq i \leq p$. Moreover for any $n \in \mathcal{N}$ sufficiently large, we have $\operatorname{rk} L^{(n)}=\operatorname{dim} \mathcal{F}=p$ and Eq. (2.1) shows that the last $q-p$ rows of $L^{(n)}$ are linear combinations of the first $p$ rows. Therefore the first $p$ rows are linearly independent: the matrix $\left[\ell_{k, i}^{(n)}\right]_{1 \leq i \leq p, 1 \leq k \leq K}$ has rank $p$. Accordingly for each $n$ there exist pairwise distinct 
integers $k_{1}, \ldots, k_{p}$ between 1 and $K$ such that the matrix $M^{(n)}=\left[\ell_{k_{j}, i}^{(n)}\right]_{1 \leq i, j \leq p}$ is invertible. Using Eq. (2.2) we may apply the usual version of Siegel's criterion (namely [9, Theorem 4]) to this matrix and deduce that

$$
\operatorname{dim}_{\mathbb{Q}} \operatorname{Span}_{\mathbb{Q}}\left(\theta_{1}^{\prime}, \ldots, \theta_{p}^{\prime}\right) \geq \tau+1 .
$$

Since $\theta_{i}^{\prime} \in \operatorname{Span}_{\mathbb{Q}}\left(\theta_{1}, \ldots, \theta_{q}\right)$ for any $1 \leq i \leq p$, this concludes the proof of Proposition 2 .

Remark 4. The idea of applying the usual version of Siegel's criterion to numbers $\theta_{i}^{\prime}$ defined as linear combinations of $\theta_{1}, \ldots, \theta_{q}$ appears also in [9] (see Proposition 2 in $\S 6$ and Eq. (9.1)). However the situation is different in that paper: the rows of the matrix $P$ (see Step 2 in $\$ 1.4$ above) are linearly dependent, which is not the case here.

\section{Deduction of Theorems 1 and 2 from Proposition 1}

In this section we prove Theorems 1 and 2 stated in the introduction, and also a result that nearly contains both of them (namely Theorem 4 stated at the end of §3.2). At last, we show in $§ 3.3$ that the linear forms constructed in [11] are a special case of those studied here.

\subsection{Proof of Theorem 1}

Let $f, T, p, \varepsilon$, and $a$ be as in the statement of Theorem 1 ; put $N=T$. We consider the complex numbers $\theta_{1}, \ldots, \theta_{a+N-1}$ given by:

$$
\left\{\begin{array}{l}
\theta_{i-1}=2(-1)^{p} L(f, i) \text { for } 2 \leq i \leq a \text { with } i \equiv p \bmod 2 \\
\theta_{i-1}=0 \text { for } 2 \leq i \leq a \text { with } i \not \equiv p \bmod 2 \\
\theta_{a+i}=f(i) \text { for } 0 \leq i \leq N-1
\end{array}\right.
$$

We apply Proposition 1 to each integer multiple $n$ of $N$, and let $\ell_{k, i}^{(n)}=s_{k, i+1}$ for $1 \leq i \leq$ $a+N-1$ and $1 \leq k \leq c_{1}$. Then we apply Siegel's linear independence criterion (namely Proposition 2 stated and proved in $\S 2$ ) with $q=a+N-1, Q_{n}=\beta^{n}$ and $\tau=-\frac{\log \alpha}{\log \beta}$ (so that $Q_{n}^{-\tau}=\alpha^{n}$ ), where $\alpha$ and $\beta$ are defined in $\S 1.1$; we take for $\mathcal{N}$ the set of integer multiples of $N$. Therefore we obtain

$$
\operatorname{dim}_{\mathbb{Q}} \operatorname{Span}_{\mathbb{Q}}(\{L(f, i), 2 \leq i \leq a \text { and } i \equiv p \bmod 2\} \cup\{f(0), \ldots, f(N-1)\}) \geq 1-\frac{\log \alpha}{\log \beta} .
$$

Taking $a$ very large and $r$ equal to the integer part of $\frac{a}{(\log (a))^{2}}$ concludes the proof of Theorem 1 since

$$
1-\frac{\log \alpha}{\log \beta}-N=\frac{1+\varepsilon_{a}}{1+\log 2} \log a \text { where } \lim _{a \rightarrow+\infty} \varepsilon_{a}=0 ;
$$

here the shift of $N$ in the lower bound comes from $f(0), \ldots, f(N-1)$ that appear in Eq. (3.1). 


\subsection{Proof of Theorem 2}

Let $f, T, E$, and $p$ be as in the statement of Theorem 2. Let $0<\varepsilon<1 / 4$ and $a$ be sufficiently large with respect to $\varepsilon, T$, and $\operatorname{dim} E$. We denote by $D$ the product of all primes less than or equal to $(1-3 \varepsilon) \log a$ (such a product has asymptotically the largest possible number of divisors with respect to its size, see [13, Chapter XVIII, §1]). Then we have

$$
\log D=\sum_{p \leq(1-3 \varepsilon) \log a} \log p \leq(1-2 \varepsilon) \log a
$$

by the prime number theorem, i.e., $D \leq a^{1-2 \varepsilon}$. We take for $r$ the integer part of $a^{\varepsilon}$. At last, we let $N=D T$.

For any divisor $d$ of $D=N / T$ and any $m \in \mathbb{Z}$, let $g_{d}(m)=f(m / d)$ if $d$ divides $m$, and $g_{d}(m)=0$ otherwise. Since $f$ is $T$-periodic we have $g_{d}(m+N)=g_{d}(m)$ for any $m$.

We shall choose below an integer $w_{d}$ for each divisor $d$ of $D$; let

$$
g=\sum_{d \mid D} w_{d} g_{D / d}
$$

We shall apply Proposition 1 to the $N$-periodic function $g$ and obtain linear forms in the numbers

$$
L(g, i)=\sum_{m=1}^{\infty} \frac{1}{m^{i}} \sum_{\substack{d|D \\ D| m d}} w_{d} f(m d / D)=\sum_{d \mid D} w_{d} \sum_{m^{\prime} \geq 1} \frac{f\left(m^{\prime}\right) d^{i}}{m^{\prime i} D^{i}}
$$

by letting $m^{\prime}=m d / D$. Therefore we have

$$
L(g, i)=D^{-i}\left(\sum_{d \mid D} w_{d} d^{i}\right) L(f, i) .
$$

Notice that $D$ has $\delta=2^{\pi((1-3 \varepsilon) \log a)}$ divisors, with

$$
\log \delta=\pi((1-3 \varepsilon) \log a) \log 2 \geq(1-4 \varepsilon)(\log 2) \frac{\log a}{\log \log a} .
$$

Assume that the number of values (0.2) which do not belong to $E$ is less than $\delta$. Let $2 \leq i_{1}<i_{2}<\ldots<i_{\delta-1} \leq a$ be integers such that if $L(f, i) \notin E$ and $i \equiv p \bmod 2$, $2 \leq i \leq a$, then $i=i_{j}$ for some $j$.

The homogeneous linear system

$$
\sum_{d \mid D} w_{d} d^{i_{j}}=0 \text { for any } j \in\{1, \ldots, \delta-1\}
$$

has $\delta$ unknowns $w_{d}$, where $d$ ranges through the set $\mathcal{D}$ of divisors of $D$, and $\delta-1$ equations. Therefore it has a non-zero integer solution $\left(w_{d}\right) \in \mathbb{Z}^{\mathcal{D}}$. 
At this point, the integers $w_{d}$ are chosen in [11] such that $\sum_{d \mid D} w_{d} d \neq 0$, using an elementary zero estimate (namely, a generalized Vandermonde determinant is non-zero). Here we do not need to make any such assumption: we just assume that $w_{d} \neq 0$ for at least one $d$. Indeed a (much more complicated) zero estimate is used in the present proof, namely Theorem 3.

Proposition 1 applies to the $N$-periodic function $g=\sum_{d \mid D} w_{d} g_{D / d}$ defined above. Using also Siegel's linear independence criterion as in $\S 3.1$ we obtain

$$
\operatorname{dim}_{\mathbb{Q}} \operatorname{Span}_{\mathbb{Q}}(\{g(0), \ldots, g(N-1)\} \cup\{L(g, i), 2 \leq i \leq a \text { and } i \equiv p \bmod 2\}) \geq 1-\frac{\log \alpha}{\log \beta}
$$

with

$$
1-\frac{\log \alpha}{\log \beta} \sim \frac{\log r}{1+\log 2} \sim \frac{\varepsilon}{1+\log 2} \log a
$$

as $a \rightarrow \infty$ (recall that $r$ is the integer part of $a^{\varepsilon}$ ).

On the other hand, the numbers that appear in the left hand side of (3.5) have the following properties:

- $g(0), \ldots, g(N-1)$ belong to $\{0, f(0), f(1), \ldots, f(T-1)\}$.

- For $2 \leq i \leq a$ with $i \equiv p \bmod 2, L(g, i)$ is zero if $i \in\left\{i_{1}, \ldots, i_{\delta-1}\right\}$, and belongs to $E$ otherwise, as Eqns. (3.2) and (3.4) show.

Therefore we have

$$
\operatorname{dim}_{\mathbb{Q}} \operatorname{Span}_{\mathbb{Q}}(\{g(0), \ldots, g(N-1)\} \cup\{L(g, i), 2 \leq i \leq a \text { and } i \equiv p \bmod 2\}) \leq T+\operatorname{dim} E .
$$

Combining Eqns. (3.5) and (3.6) yields a contradiction provided $a$ is large enough. This concludes the proof of Theorem 2 .

Since $4(1+\log 2)>7$, the same proof (with $\varepsilon$ replaced with $\varepsilon / 4$ to take Eq. (3.3) into account) provides the following refinement of Theorem 2.

Theorem 4. Let $T \geq 1$, and $f: \mathbb{Z} \rightarrow \mathbb{C}$ be such that $f(n+T)=f(n)$ for any $n$. Assume that $f$ is not identically zero. Let $p \in\{0,1\}, 0<\varepsilon<1$, and a be sufficiently large (in terms of $T$ and $\varepsilon)$. Let $E$ be a finite-dimensional $\mathbb{Q}$-vector space contained in $\mathbb{C}$ with $\operatorname{dim} E<\frac{\varepsilon}{7} \log a$. Then among the numbers $L(f, s)$ with $2 \leq s \leq a$ and $s \equiv p \bmod 2$, at least

$$
2^{(1-\varepsilon) \frac{\log a}{\log \log a}}
$$

do not belong to $E$.

Choosing $\varepsilon=7 / 8$, this refinement implies that the numbers $L(f, s)$ with $2 \leq s \leq a$ and $s \equiv p \bmod 2$ are not all contained in such a subspace $E$ : they span a $\mathbb{Q}$-vector space of dimension at least $\frac{1}{8} \log a$. Except for the multiplicative constant $\left(\frac{1}{8}\right.$ instead of $\left.\frac{1-o(1)}{1+\log 2}\right)$, Theorem 1 follows as a corollary of Theorem 4 . 


\subsection{Connection to the proof of $[11]$}

In this section we show that the linear forms used in [11] to prove (0.1) are a special case of those studied in the present paper (namely in the proof of Theorem 2 with $f(m)=1$, $T=r=k=1$, and $p \equiv a \bmod 2$ ). Accordingly they are related to the Padé approximation problem stated in $\S 1.4$, in which the number of equations is essentially equal to the number of unknowns.

We keep the notation of the proof of Theorem 2 in $\S 3.2$, with $T=1$ and $f(m)=m$ for any $m \in \mathbb{Z}$. In particular $N=D$ is the product of all primes less than or equal to $(1-3 \varepsilon) \log a$. For any divisor $d$ of $D$ we have $g_{d}(m)=1$ if $d$ divides $m$, and $g_{d}(m)=0$ otherwise. The function $g=\sum_{d \mid D} w_{d} g_{D / d}$ satisfies

$$
g(m)=\sum_{\substack{d|D \\ D| m d}} w_{d} \text { for any } m \in \mathbb{Z} .
$$

Now let $n$ be an integer multiple of $2 N=2 D$, and let $p \in\{0,1\}$ be such that $p \equiv a \bmod 2$. Then the rational function $F$ satisfies the symmetry property of well-poised hypergeometric series:

$$
F(-n-t)=(-1)^{(2 r+1) n+1+(a+1)\left(\frac{n}{N}+1\right)} F(t)=(-1)^{p} F(t) .
$$

This is the key ingredient (since the Ball-Rivoal theorem) to get rid of even zeta values, when $p=1$. In our approach where Nesterenko's linear independence criterion is replaced with Siegel's combined with Shidlovsky's lemma, this property cannot be used in the same way because it is destroyed when considering $S^{(k-1)}(z)$ for $k \geq 2$. Using both $S_{0}$ and $S_{\infty}$ in constructing the linear forms (see $§ 1.2$ ) makes it possible to overcome this difficulty (as in [8]). With $k=1$ this trick does not modify the linear forms we are interested in, since for any $\ell \in \mathbb{Z}$ we have using Eqns. (1.3) and (3.8) and the fact that $N$ divides $n$ :

$$
\begin{aligned}
S_{0}\left(\omega^{\ell}\right)+(-1)^{p} S_{\infty}\left(\omega^{-\ell}\right) & =\sum_{t=n+1}^{\infty} F(-t) \omega^{\ell t}+(-1)^{p} \sum_{t=1}^{\infty} F(t) \omega^{\ell t} \\
& =\sum_{t=1}^{\infty} \omega^{\ell t}\left(F(-n-t)+(-1)^{p} F(t)\right) \\
& =2(-1)^{p} \sum_{t=1}^{\infty} F(t) \omega^{\ell t} .
\end{aligned}
$$

We are now in position to express differently the linear forms constructed in part (iii) of Proposition 1 from the map $g$ given by Eq. (3.7), in the special case where $N=D, n$ is a multiple of $2 N, p \equiv a \bmod 2, r=1$, and $k=1$. Denote by $\Lambda_{n}$ this linear form. Then 
we have using Lemma 1 and Eqns. (3.9) and (3.7):

$$
\begin{aligned}
\delta_{n}^{-1} \Lambda_{n} & =\sum_{\ell=1}^{D} \widehat{g}(\ell)\left(S_{0}\left(\omega^{\ell}\right)+(-1)^{p} S_{\infty}\left(\omega^{-\ell}\right)\right) \\
& =2(-1)^{p} \sum_{t=1}^{\infty} F(t) \sum_{\ell=1}^{D} \widehat{g}(\ell) \omega^{\ell t} \\
& =2(-1)^{p} \sum_{t=1}^{\infty} F(t) g(t) \\
& =2(-1)^{p} \sum_{d \mid D} w_{d} \sum_{t \geq 1} F(t) \\
& =2(-1)^{p} \sum_{d \mid D} w_{d} \sum_{t^{\prime}=1}^{\infty} F\left(D t^{\prime} / d\right) \\
& =2(-1)^{p} \sum_{d \mid D} w_{d} \sum_{j=1}^{d} \sum_{m=1}^{\infty} F\left(m D+j \frac{D}{d}\right) .
\end{aligned}
$$

In the last expression the sum on $m \geq 1$ should have begun at $m=0$, but this makes no difference since $F(j D / d)=0$ for any $1 \leq j \leq d$. Now let $R(t)=F(D t)$; then we have

$$
\frac{(-1)^{p}}{2 \delta_{n}} \Lambda_{n}=\sum_{d \mid D} w_{d} \sum_{j=1}^{d} \sum_{m=1}^{\infty} R\left(m+\frac{j}{d}\right) .
$$

Up to the normalizing factor $\frac{(-1)^{p}}{2 \delta_{n}}$ these are exactly the linear forms $\widehat{r_{n}}$ used in [11] to prove (0.1). Indeed the following notation is used in [11] for $1 \leq j \leq D$ and $d \mid D$ :

$$
\begin{gathered}
R_{n}(t)=D^{3 D n} n !^{s+1-3 D} \frac{\prod_{j=0}^{3 D n}\left(t-n+\frac{j}{D}\right)}{\prod_{j=0}^{n}(t+j)^{a+1}}, \quad r_{n, j}=\sum_{m=1}^{\infty} R_{n}\left(m+\frac{j}{D}\right), \\
\widehat{r}_{n, d}=\sum_{j=1}^{d} r_{n, j \frac{D}{d}}, \quad \widetilde{r}_{n}=\sum_{d \mid D} w_{d} \widehat{r}_{n, d} .
\end{gathered}
$$

Now, up to the normalizing factor $R_{n}(t)$ is equal to the rational function $R(t)=F(D t)$ so that $\Lambda_{n}$ is equal to $\widetilde{r}_{n}$ using Eq. (3.10).

\section{References}

[1] R. ApÉRY - "Irrationalité de $\zeta(2)$ et $\zeta(3)$ ", in Journées Arithmétiques (Luminy, 1978), Astérisque, no. 61, 1979, p. 11-13. 
[2] K. Ball \& T. Rivoal - "Irrationalité d'une infinité de valeurs de la fonction zêta aux entiers impairs", Invent. Math. 146 (2001), no. 1, p. 193-207.

[3] D. Bertrand - "Le théorème de Siegel-Shidlovsky revisité", in Number theory, Analysis and Geometry: in memory of Serge Lang (D. Goldfeld et al., eds.), Springer, 2012, p. 51-67.

[4] D. Bertrand \& F. Beukers - "Équations différentielles linéaires et majorations de multiplicités", Ann. Sci. École Norm. Sup. (4) 18 (1985), no. 1, p. 181-192.

[5] P. Colmez - "Arithmétique de la fonction zêta", in Journées mathématiques X-UPS 2002, éditions de l'école Polytechnique, 2003, p. 37-164.

[6] N. Fel'dman \& Y. Nesterenko - Number theory IV, transcendental numbers, Encyclopaedia of Mathematical Sciences, no. 44, Springer, 1998, A.N. Parshin and I.R. Shafarevich, eds.

[7] S. Fischler - "Distribution of irrational zeta values", Bull. Soc. Math. France 145 (2017), no. 3, p. 381-409.

[8] — , "Shidlovsky's multiplicity estimate and irrationality of zeta values", J. Austral. Math. Soc. 105 (2018), no. 2, p. 145-172.

[9] S. Fischler \& T. RIVOAL - "Linear independence of values of G-functions, II. Outside the disk of convergence", preprint arXiv 1811.08758 [math.NT], soumis.

[10] — , "Approximants de Padé et séries hypergéométriques équilibrées", J. Math. Pures Appl. 82 (2003), no. 10, p. 1369-1394.

[11] S. Fischler, J. Sprang \& W. Zudilin - "Many odd zeta values are irrational", preprint arXiv 1803.08905 [math.NT], Compositio Math., to appear, 2018.

[12] S. Gun, M. R. Murty \& P. Rath - "On a conjecture of Chowla and Milnor", Canad. J. Math. 63 (2011), no. 6, p. 1328-1344.

[13] G. Hardy \& E. Wright - An introduction to the theory of numbers, fifth éd., Oxford Science Publications, 1979.

[14] T. Hessami Pilehrood \& K. Hessami Pilehrood - "Irrationality of sums of zeta values", Mat. Zametki [Math. Notes] 79 (2006), no. 4, p. 607-618 [561-571].

[15] R. Marcovecchio - "Linear independence of linear forms in polylogarithms", $A n$ nali Scuola Norm. Sup. Pisa V (2006), no. 1, p. 1-11.

[16] T. Matala-Aho - "On Diophantine approximations of the solutions of $q$-functional equations", Proc. Roy. Soc. Edinburgh Sect. A 132 (2002), p. 639-659. 
[17] M. H. NASH - "Special values of Hurwitz zeta functions and Dirichlet $L$-functions", Ph.D. thesis, Univ. of Georgia, Athens, U.S.A., 2004.

[18] J. NeUKIRCH - Algebraic number theory, Springer, 1999.

[19] M. Nishimoto - "On the linear independence of the special values of a Dirichlet series with periodic coefficients", preprint arXiv 1102.3247 [math.NT], 2011.

[20] T. RivoAL - "La fonction zêta de Riemann prend une infinité de valeurs irrationnelles aux entiers impairs", C. R. Acad. Sci. Paris, Ser. I 331 (2000), no. 4, p. 267-270.

[21] T. Rivoal \& W. Zudilin - "Diophantine properties of numbers related to Catalan's constant", Math. Annalen 326 (2003), no. 4, p. 705-721.

[22] A. B. Shidlovsky - Transcendental numbers, de Gruyter Studies in Math., no. 12, de Gruyter, Berlin, 1989.

[23] J. Sprang - "Infinitely many odd zeta values are irrational. By elementary means", preprint arXiv:1802.09410 [math.NT], 2018.

[24] W. Zudilin - "One of the odd zeta values from $\zeta(5)$ to $\zeta(25)$ is irrational. By elementary means", SIGMA 14 (2018), no. 028, 8 pages. 